\title{
Investigation of Induction Period, Spectral, Band Gap Energy, Microhardness and Optical Property of Thiosemicarbazone of Acetaldehyde
}

\author{
G. V. PANDIAN ${ }^{1}$ and P. ANBUSRINIVASAN ${ }^{2 *}$ \\ ${ }^{1}$ Department of Chemistry, T.B.M.L College, Porayar, Nagapattinam Dt, Tamilnadu, India \\ ${ }^{2}$ Department of Chemistry, AVC College(Autonomous), Mayiladuthurai,, Tamilnadu, India \\ gvpsjatbmlc@gmail.com
}

Received 11 May 2014 / Accepted 2 June 2014

\begin{abstract}
An organic crystal of Thiosemicarbazone of acetaldehyde was grown by adopting slow evaporation solution growth technique (SESGT) using methanol as a solvent. The crystal dimension up to $10 \times 4 \times 6 \mathrm{~mm}^{3}$ was obtained. The harvested crystal were purified by repeated recrystallization. The induction period was measured at various super saturations. These crystals were characterized by FT-IR spectra to identify functional groups. The molecular structure was analyzed by ${ }^{1} \mathrm{H}$ NMR. The semiconducting property was calculated by band gap energy calculation. Vickers microhardness studies provides information about the hardness of the grown crystal. Second harmonic generation efficiency of the powdered thiosemicarbazone of acetaldehyde was tested using Nd:YAG laser and it is found to be 1.6 times higher than that of potassium dihydrogen phosphate.
\end{abstract}

Keywords: Slow evaporation, Solution growth technique, Induction period, Band gap energy, Microhardness, SHG efficiency.

\section{Introduction}

Recent researches have mentioned that organic crystals are bulk in size, hard, stable and large nonlinear optical susceptibilities compared to the inorganic crystals but they have poor mechanical properties. Organic nonlinear crystals possess shorter wavelength, optical quality sufficiently larger nonlinear coefficient. Considering all these parameters, the modern scientists have concentrated on the growth of organic crystals ${ }^{1,2}$. An organic crystal of thiosemicarbazone of acetaldehyde play an important role in application of optical computing and optical communication devices and optical communication processes. These crystals were prepared by slow evaporation solution growth technique (SESGT). The grown crystals were characterized by FT-IR spectral analysis, UV-Visible, ${ }^{1} \mathrm{H}$ NMR, band gap energy and microhardness studies ${ }^{3-5}$. The induction period for solution grown thiosemicarbazone of acetaldehyde were determined and reported for the first time. 


\section{Experimental}

To a hot solution of Thiosemicarbazide in an appropriate amount of methanol was added drop wise to a solution of the acetaldehyde in needed quantity of methanol during thirty minutes. The mixture was thoroughly stirred and refluxed for four hours. Then it was filtered and the filtrate was concentrated to half the volume under reduced pressure ${ }^{6,7}$. Then the concentrate was allowed for slow evaporation at room temperature. The crystals were collected by filtration, washed with cold ethanol and dried in vacuo. The harvested crystals are shown in Figure 1. These crystals are suitable for characterization studies.

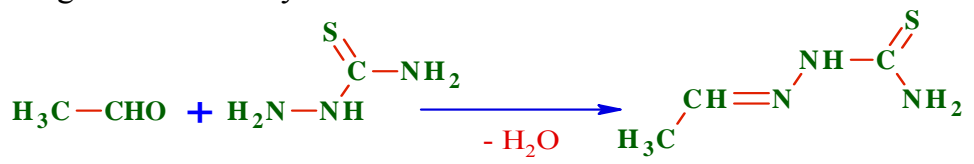

Acetaldehyde Thiosemicarbazide Thiosemicarbazone of acetaldehyde

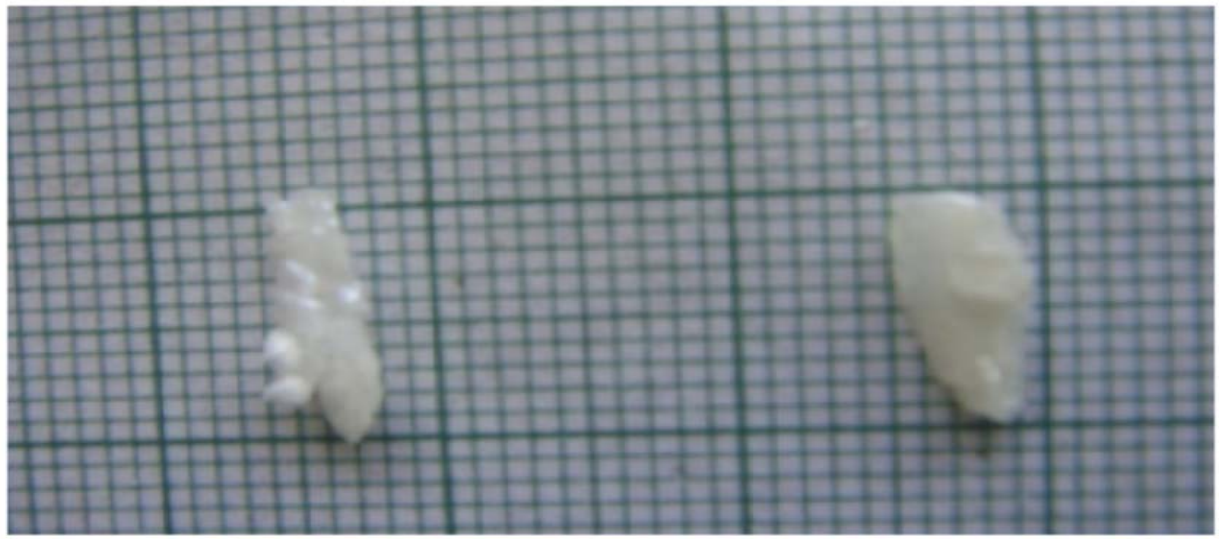

Figure 1. Photograph of thiosemicarbazone of acetaldehyde

\section{Experimental determination of induction period and interfacial energies}

There are several methods of measuring the induction period depending upon the solubility of the materials. Here the visual observation method was followed. Solutions of Thiosemicarbazone of acetaldehyde in methanol at different super saturation values were prepared and subjected to systematic slow evaporation. The time period that elapses between the achievement of super saturation and appearance of visual nuclei is taken as the induction period $(\tau)$. Several trial runs were performed to minimize the error. Experiments were repeated for super saturation(s) like $1.15,1.17,1.20$ and 1.25 at two different temperatures. From the results obtained a plot of $\ln \tau$ against $1 /(\operatorname{lns})^{2}$ was drawn There are several methods of measuring the induction period depending upon the solubility of the materials ${ }^{8-11}$. Here the visual observation method was followed. Solutions of thiosemicarbazone of acetaldehyde in methanol at different super saturation values were prepared and subjected to systematic slow evaporation. The time period that elapses between the achievement of super saturation and appearance of visual nuclei is taken as the induction period $(\tau)$. Several trial runs were performed to minimize the error. Experiments were repeated for super saturation(s) like 1.15, 1.17, 1.20 and is shown in Figure 2. The interfacial tension was calculated from the slope of the curves using the equation

$$
\ln \tau=\ln A+16 \pi r^{3} V^{2} N / 3 R T(\ln s)^{2}
$$


Where ' $A$ ' is a constant related to the pre-exponential factor of the nucleation rate expression. ' $V$ ' is the molar volume, ' $N$ ' is the Avogadro number and ' $R$ ' is the gas constant. The factor $16 \pi \mathrm{r}^{3}$ in the above equation refers to the spherical nuclei. The interfacial tension between the thiosemicarbazone of acetaldehyde and methanol is calculated by measuring the slope value of the curve obtained at the two temperatures.

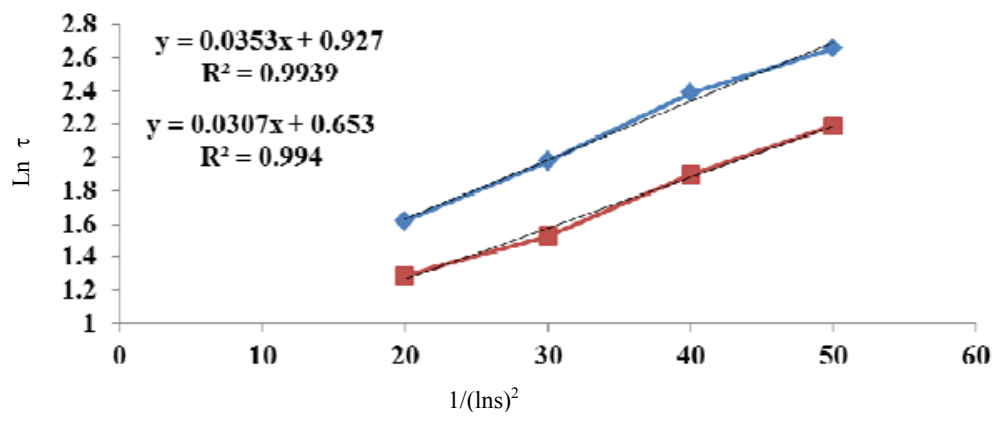

Figure 2. A Plot of $\ln \tau$ vs. $(\ln s)^{2}$ for thiosemicarbazone of acetaldehyde grown from methanol at 308 and 313

\section{Results and Discussion}

FT-IR Spectral studies

Fourier transform infrared (FT-IR) spectrum is an important record, which gives sufficient information about the structure of a compound. In this technique almost all functional groups in a molecule absorb characteristically within a definite range of frequency ${ }^{12-14}$. The absorption of infrared radiation causes the various bands in a molecule to stretch and bend with respect to one another. The FT-IR spectral studies of solution grown Thiosemicarbazone of acetaldehyde was recorded using $\mathrm{KBr}$ pellet technique between $400 \mathrm{~cm}^{-1}$ and $4000 \mathrm{~cm}^{-1}$. The FT-IR spectrum is shown in Figure 3. The peak at $1531.91 \mathrm{~cm}^{-1}$ shows corresponding imine group $(\mathrm{C}=\mathrm{N})$ which confirm the bond between Aldehyde and Hydrazide is imine group. The peak at $3366 \mathrm{~cm}^{-1}$ shows N-H stretching vibration. The peak below $1485 \mathrm{~cm}^{-1}$ indicates $\mathrm{C}=\mathrm{N}$ and N-N stretching vibration. The $\mathrm{C}-\mathrm{H}$ stretching vibration shown by a weak absorption at $3177 \mathrm{~cm}^{-1}$. The $\mathrm{C}=\mathrm{S}$ stretch of Thiosemicarbazide moiety is observed at $1163 \mathrm{~cm}^{-1}$. The absence of band at $2720 \mathrm{~cm}^{-1}$ indicates the absence of aldehyde function. The absence of peak at $2700 \mathrm{~cm}^{-1}$ confirms the absence of aldehyde functional group in thiosemicarbazone of acetaldehyde.

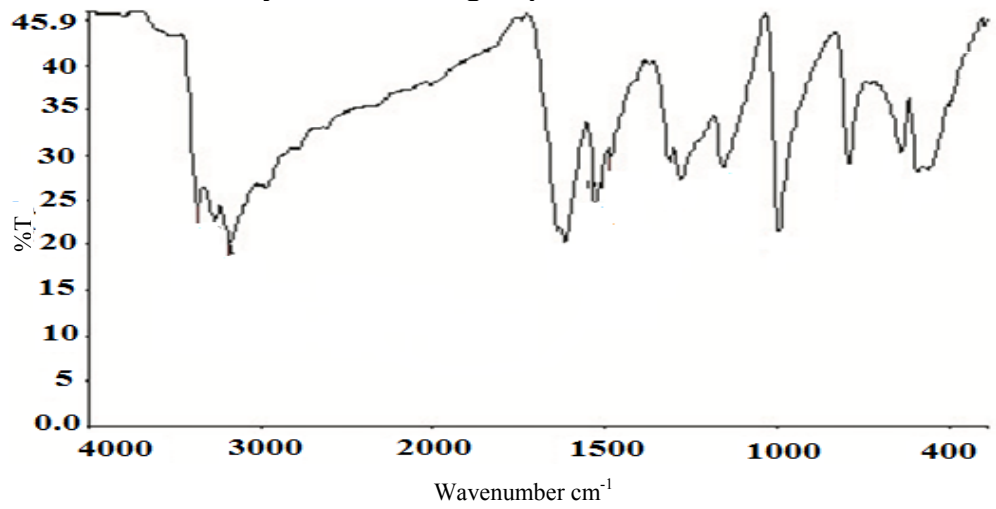

Figure 3. FT-IR Spectrum of thiosemicarbazone acetaldehyde 


\section{UV Visible spectral studies}

UV-Visible spectral study is very useful technique to determine the transparency of a substance. The UV- Visible spectrum of thiosemicarbazone of acetaldehyde crystal was recorded using Lambda 25 Spectrometer. The UV-Visible spectrum of thiosemicarbazone of acetaldehyde is shown in Figure 4. The characteristic absorption of thiosemicarbazone of acetaldehyde crystal is found between $260-320 \mathrm{~nm}$. The spectrum further shows the highly transparent nature of the thiosemicarbazone of acetaldehyde between $320-800 \mathrm{~nm}$ which is one of the important characteristic properties of a material suitable for optical applications ${ }^{15}$.

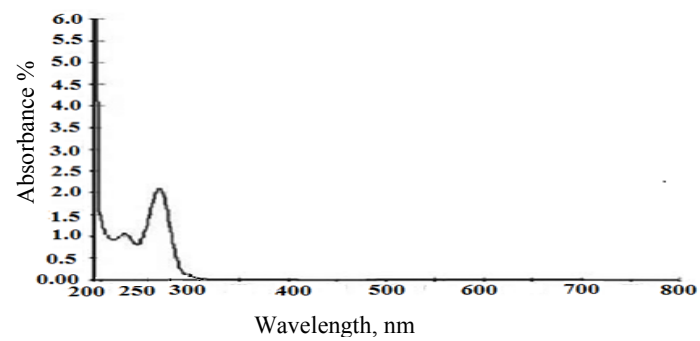

Figure 4. UV- Visible spectrum of thiosemicarbazone acetaldehyde

\section{NMR Spectral analysis}

\section{${ }^{1} H$ NMR Spectral analysis}

The Nuclear Magnetic Resonance Spectral analysis is useful in the determination of the molecular structure based on the chemical environment of the magnetic nuclei such as ${ }^{1} \mathrm{H},{ }^{13} \mathrm{C}$, ${ }^{31} \mathrm{P}$ etc., even at low concentrations ${ }^{16}$. The proton NMR spectral analysis of thiosemicarbazone of acetaldehyde was carried out using BRUKER $300 \mathrm{NMR}$ spectrometer at $300 \mathrm{MHz}$ using DMSO as solvent. The recorded proton nuclear magnetic resonance spectrum of thiosemicarbazone of acetaldehyde is shown in Figure 5. In this spectrum a peak observed at $\delta=2.502 \mathrm{ppm}$ is corresponding to methyl group. The $\mathrm{NH}_{2}$ protons of hydrazide part is observed at $\delta=8.624 \mathrm{ppm}$ as a broad singlet. The $-\mathrm{NH}$ proton is observed at $\delta=7.2 \mathrm{ppm}$. The $\mathrm{CH}$ proton is observed at $\delta=4.484 \mathrm{ppm}$. The HOD signals is observed at $\delta=3.413 \mathrm{ppm}$. The Residual protons of solvent DMSOd6 is observed at $\delta=2.498 \mathrm{ppm}$. The formation of hydrazide is confirmed by NMR spectral analysis. The spectral data obtained for the thiosemicarbazone of acetaldehyde are well in accordance with theoretical and standard spectrum.

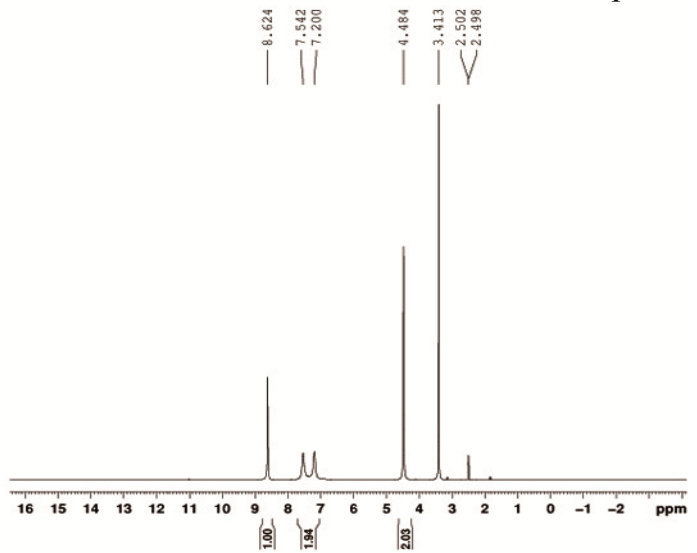

Figure 5. ${ }^{1}$ H NMR Spectrum of thiosemicarbazone of acetaldehyde 


\section{Band gap energy calculation}

Band gap refers to the energy difference (in electron volts) between the top of the valence band and the bottom of the conduction band in insulators and semiconductors. This is equivalent to the energy required to free an outer shell electron from its orbit about the nucleus to become a mobile charge carrier, able to move freely within the solid material, so the band gap is a major factor determining the electrical conductivity of a solid .The band gap energy of prepared thiosemicarbazone of acetaldehyde crystal is evaluated from the relation between absorption coefficient $\alpha$ and photon energy $h v^{17}$.

$$
(\alpha h v)=A\left(h v-E_{g}\right)^{x}
$$

Where $\mathrm{A}$ is a constant, $\mathrm{E}_{\mathrm{g}}$ is the band gap and $x=1 / 2$ for directly allowed electronic transitions. Figure 6 shows the plot between $(\alpha h v)^{2}$ and $h v$ of the prepared thiosemicarbazone of acetaldehyde crystal. The extrapolation of linear portion of the curves on $h v$ axis gives the direct band gap energy. The value of band gap energy for prepared thiosemicarbazone of acetaldehyde crystal is $3.991 \mathrm{eV}$. It revealed that the grown thiosemicarbazone of acetaldehyde crystal has semiconducting property

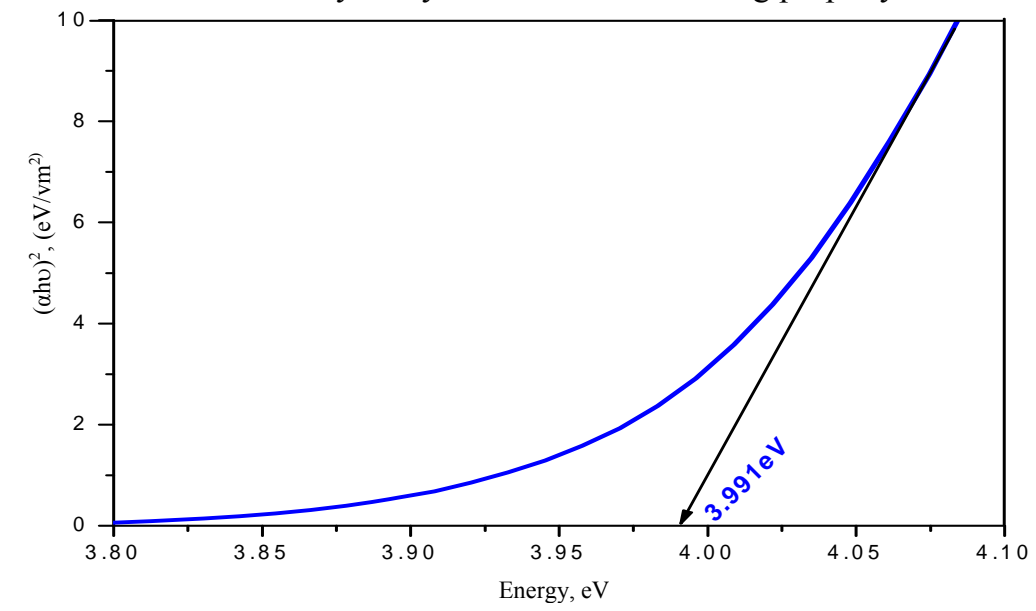

Figure 6. Band gap energy spectrum of thiosemicarbazone of acetaldehyde

\section{Microhardness analysis}

Measurement of hardness is a useful nondestructive testing method to determine the hardness of the materials. The microhardness value correlates with other mechanical properties such as elastic constants and yield strength . The hardness of a material depends on different parameters such as lattice energy, Debye temperature, heat of formation and interatomic spacing ${ }^{18}$. Vickers microhardness test was carried out on thiosemicarbazone of acetaldehyde crystal using microhardness tester fitted with a diamond indenter. The indentations were made using a Vickers pyramidal indenter for various loads. The diagonals of the impressions were measured using Shimadzu, Model HMV-2 hardness instrument.

The average diagonal length of the indented impression was calculated and the Vickers microhardness number $(H v)$ was found from the relation $H v=2 P \sin (\theta / 2) / d^{2}$. If $p$ is the applied load $(\mathrm{kg})$ and $\mathrm{d}$ is the average diagonal length of the indentation impressions $(\mathrm{mm})$ and the angle between the opposite faces of the diamond pyramid is $\theta=136^{\circ}$ then $\mathrm{Hv}$ in $\mathrm{kg} / \mathrm{mm}$ is given by:

$$
\mathrm{Hv}=1.8544\left(\mathrm{P} / \mathrm{d}^{2}\right) \mathrm{kg} / \mathrm{mm}^{2}
$$

where, 1.8544 is a constant of a geometrical factor for the diamond pyramid 
The variation of microhardness values with applied load is shown in Figure 7. From the Vickers microhardness studies it observed that the hardness values increases up to load of $50 \mathrm{~g}$. For load above $50 \mathrm{~g}$ crack started developing around the indentation mark which may be due to the release of internal stresses. The presence of cracks confirms the decrease in microhardness

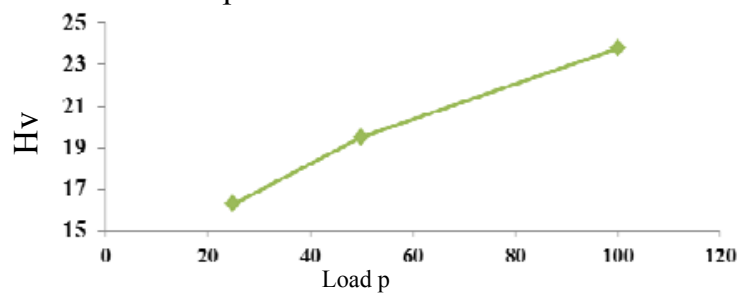

Figure 7. Microhardness values vs. load for thiosemicarbazone of acetaldehyde

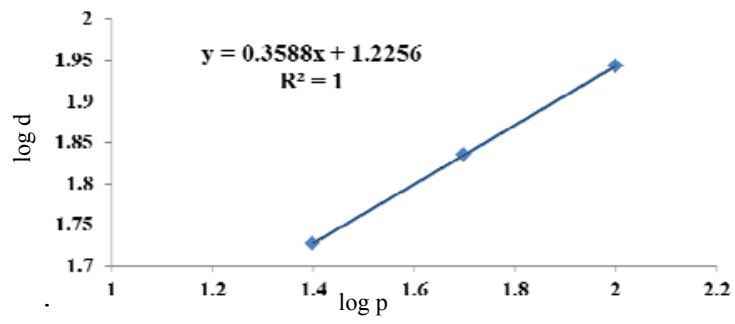

Figure 8. The plot of $\log p$ vs. $\log d$

Work hardening coefficient $\mathrm{n}$, a measure of the strength of the crystal is computed from the $\log p$ - $\log d$ plot (Figure 8) and it is found to be 3.5.

\section{Nonlinear optical studies}

Kurts and perry second harmonic generation (SHG) tests ${ }^{19}$ was performed to determine the NLO efficiency of thiosemicarbazone of acetaldehyde crystal. The grown crystal was powdered with a uniform particle size and packed in a micro capillary of uniform bore and was illuminated using spectra physics quanta ray DHS2.Nd: YAG laser is used to test second harmonic generation (SHG) of grown crystal, The SHG efficiency obtained for thiosemicarbazone of acetaldehyde is found to be about 1.6 times higher than that of potassium dihydrogen orthophosphate crystal.

\section{Conclusion}

The induction period at various super saturations were determined and reported for the first time. An optically good grade thiosemicarbazone of acetaldehyde crystal were successfully prepared and grown by adopting slow evaporation solution growth technique, using methanol as a solvent. The FT-IR spectral report confirms the purity and presence of functional group. The characteristic absorption of thiosemicarbazone of acetaldehyde crystal is found between 260-320 nm. The spectrum further shows the highly transparent nature of the thiosemicarbazone of acetaldehyde between $320-800 \mathrm{~nm}$. The ${ }^{1} \mathrm{H}$ NMR spectral analysis also characterize the molecular structure. The value of band gap energy for grown crystal is $3.99 \mathrm{eV}$. It supports the semiconducting property of the grown thiosemicarbazone of acetaldehyde crystal. Vickers Microhardness studies shows that the hardness values increases up to load of $50 \mathrm{~g}$. The SHG efficiency obtained for thiosemicarbazone of acetaldehyde is about 1.6 times higher than that of potassium dihydrogen orthophosphate crystal. Microhardness studies, Band gap energy calculation and SHG efficiency of thiosemicarbazone of acetaldehyde may be useful in deciding the application oriented properties 


\section{Acknowledgement}

The authors wish to thank Dr. G. Jonas Gunasekaran, Dr. Thiyagesan and Dr. P. C. Jobe Prabakar for their encouragement. The authors are thankful Dr. Brinda Department of Centre for (CARISM) Sastra University, Thanjavur, India

\section{References}

1 Narayanamoolaya B and Dharmaprakash S M, J Crystal Growth, 2006, 290, 498-503; DOI: $10.1016 /$ j.jcrysgro.2006.01.061

2 Manivannan S and Danuskodi S, J Crystal Growth, 2003, 257, 305-307; DOI: 10.1016/S0022-0248(03)01466-0

3. Ramalingam S, Anbusrinivasan P and Periandy S, Spectrochimica Acta, Part A, 2011, 78, 826-834; DOI: 10.1016/j.saa.2010.12.041

4. Madhurambal G, Ramasamy P, Anbusrinivasan P, Vasudevan G, Kavitha S and Mojumdar S C, J Therm Anal Calorim., 2008, 94, 59-65; DOI: 10.1007/s10973008-9188-7

5. Madhurambal G and Anbu Srinivasan P, Cryst Res Tech., 2006, 41(3), 231-235; DOI: $10.1002 /$ crat.200510565

6. Wiliredo Hermandeza, Juanpaz, Abraham Vaisberg, Evgenia Spodine, Rainer Richter and Lothar Beyer, Bioinorgchem Appl., 2008, 2008, PMC2615113; DOI: $10.1155 / 2008 / 690952$

7. Santhakumari R and Ramamurthy K, Spectrochimica Acta part A, 2011, 78, 653659; DOI: DOI: 10.1016/j.saa.2010.11.043

8. Anbusrinivasan P, Madhurambal G and Mojumdar S C, J Therm Anal Calorim., 2012, 108, 939-946; DOI: 10.1007/s10973-012-2381-8

9. Ushasree P M, Muralidharan R, Jayavel R and Ramasamy P, J Cryst Growth, 2000, 210, 741-745; DOI: 10.1016/S0022-0248(99)00900-8

10. Kanagasekaran T, Gunasekaran M, Srinivasan P, Jayaraman D, Gopalakrishnan R and Ramasamy R, Cryst Res Tech., 2005, 40, 1128-1133;

DOI: $10.1002 /$ crat.200410504

11. Chenthamarai S, Jayaraman D, Ushasree P M, Meera K, Subramanian C and Ramasamy P, Materials Chemistry and Physics, 2000, 64, 179-183; DOI: 10.1016/S0254-0584(99)00229-1

12. Madhurambal G, Ramasamy $P$, AnbuSrinivasan $P$, Suganthi M, Vasudevan $G$ and Mojumdar S C, Asian J Chem., 2008, 20, 1775-1780.

13. Anbusrinivasan P and Kavitha S, Asian J Chem., 2008, 20, 979-982.

14. Anbusrinivasan P, Madhurambal G and Mojumdar S C, J Therm Anal Calorim., 2009, 96(1), 111-115; DOI: 10.1007/s10973-008-9882-5

15. William Kemp, Organic Spectroscopy $-3^{\text {rd }}$ Ed., 1991.

16. Dos Santos M H, Nagem T J, Braz-Filho R, ILuta I S and Speziali N L, Magn Reson Chem., 2001, 39, 155-158; DOI: 10.1002/mrc.795.

17. Fahrenbruch A L and Bube R H (Eds.), Fundamentals of Solar Cells, Academic, New York, 1993.

18. Li Hong and Bradt R C, Mater Sci Eng., A, 1991, 42, 51-61; DOI: 10.1016/09215093(91)90753-A

19. Kurtz S K and Perry T T, J Appl Phys., 1968, 39, 3798-3813; DOI: $10.1063 / 1.1656857$ 